\title{
Performance Improvement Strategies of Analog \& Mixed Circuits
}

\author{
Saumya Srivastava, Tripti Sharma
}

\begin{abstract}
In modern days technology becomes very advanced and it moves from analog to digital domain. Everything depends on one and zero frame. In any case, the foundation of any computerized circuit is constantly simple and without simple can't envision this world. ADCs play an intermediate role between analog and digital circuits. Analog and mixed circuits are basic piece of any gadget and there are bunches of works in analog domain for researcher. This paper is extremely helpful for understanding the extent of analog and mixed circuits, also give description about various smart techniques which help to improve the performance of the device. Presently gadgets turn out to be little because of scaling and circuits works on nano-scale process so area become comparably very small but on the penalty of power dissipation which is important term for consideration. This paper additionally talked about different way to deal with decrease control supply. It is thoroughly legitimizes the way that Analog is exceptionally amazing area for researcher.
\end{abstract}

Index Terms: Analog \& Mixed signal, Bulk driven, Floating gate, Slew Rate, DTMOS, DC gain

\section{INTRODUCTION}

Analog and mixed signal are basics of any device, although today technologies moves to digital side but spine of any device is analog. Analog circuits are very complex for analysis and there are lots of good challenges for new researcher. Analog domain always based on continuous signal but digital is in 0 and 1 form [1] Due to continuous nature it is difficult to work in this domain. Digital area mainly concerned about speed, how fast gadgets perform the task. In analog domain speed is considered in the form of bandwidth so analog circuit requires high bandwidth to make device faster. Noise is main issue in analog domain for high accuracy [2]. There are lots of EDA (Electronic design automation) tools are available like HSPICE, ELDOSPICE, PSPICE, TANNER, Mentor Graphics and CADENCE VIRTUOSO which helpful to enhance the performance of analog circuit based design simulation. Power supply reduction is exceptionally urgent issue in modern days. Performance parameters of analog circuits are Slew-rate, DC gain, Unity gain bandwidth and common mode rejection ratio. These factors plays important role to improve the device performance. Analog domain has many opportunities to generate new building block and improve overall performance of the device. Researcher can also work on reducing chip area of the device and try to make compact device. There are various technologies are available which are very helpful to reduce chip size, Power consumption. Bulk driven, DTMOS, FVF(Flipped voltage follower) and

Revised Manuscript Received on July 19, 2019.

Saumya Srivastava, Tripti Sharma ECED, Chandigarh University, Mohali, India

and recycling techniques help to improve the gadgets performance. Operational amplifier is basic building block of analog circuits.

\section{TECHNIQUES TO REDUCE SUPPLY VOLTAGE \& POWER SUPPLY}

\section{A. Bulk Driven MOSFET}

Bulk driven technique is an ideal way to minimize the threshold voltage and low threshold voltage is extremely valuable to reduce the power. In this technique bulk acts like gate terminal and it operates as a depletion type JFET [6]. Input signal is applied to the bulk terminal and amount of current is controlled by bulk. Device will be on small positive voltage and it will enhance ICMR. Fig-1 shows bulk driven MOSFET here bulk used as a gate terminal, it shows simple PMOS current mirror by using both gate driven and bulk driven. In gate driven current mirror's output current follows its input more closely than bulk driven MOSFET current mirror design [6].

Table-I presents a proper comparison value of different essential parameters of analog design for different SUB-1V amplifiers with bulk driven techniques. (a)

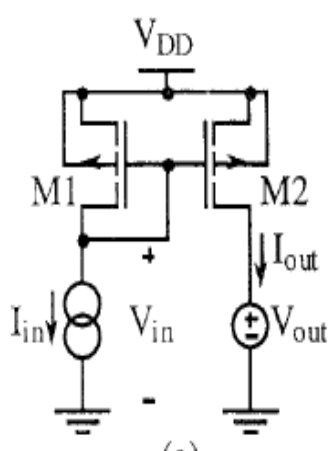

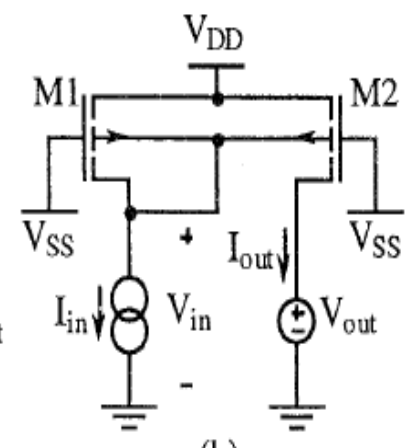

(b)
Fig-1: (a) PMOS gate driven current mirror (b) PMOS bulk driven current mirror [6]

\section{B. Floating Gate MOSFET}

Floating gate technology is based on capacitor instead of resistor based technique. In this Floating gate technique multiple inputs are deposited above the floating gate. Floating gate method is very useful to design CMOS transconductors. FGMOS is very convenience for scaling purpose and level shifting of input voltages due to capacitor divider at input stage [8]. It is beneficial for low threshold voltage and high output impedance. Due to low threshold voltage essential term power consumption is also low. FGMOS technique multiple inputs are very useful for tenability. Drawback of this technique is low bandwidth and it affects the speed of the device. Fig-2 shows how FGMOS transistors allow the implementation of compact programmable current mirrors. 


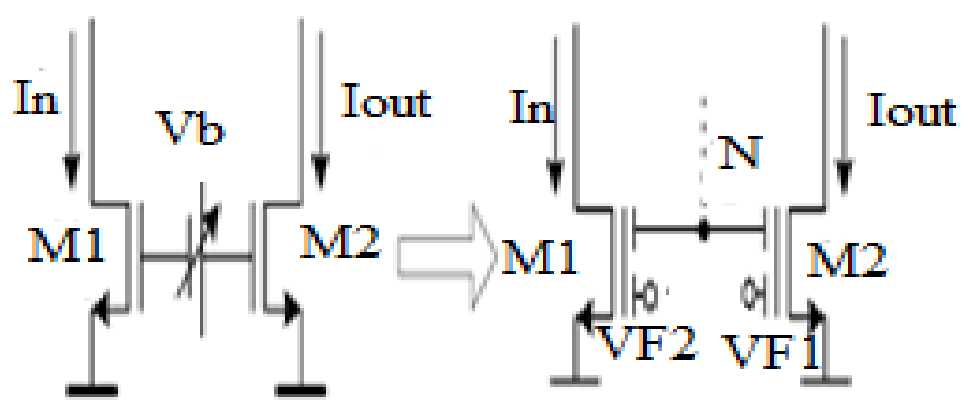

Fig-2: FGMOS Programmable current mirror [8]

Table-I Comparison between other SUB-1V amplifiers with bulk driven [7]

\begin{tabular}{|l|l|l|l|l|l|l|l|l|l|l|}
\hline Works & $\begin{array}{l}\text { Tech } \\
(\mu \mathrm{m})\end{array}$ & $\begin{array}{l}\mathrm{C}_{\mathrm{L}} \\
(\mathrm{pF})\end{array}$ & $\begin{array}{l}\text { UGBW } \\
\left(\mathrm{MH}_{\mathrm{Z}}\right)\end{array}$ & $\begin{array}{l}\text { Gain } \\
(\mathrm{db})\end{array}$ & $\begin{array}{l}\text { PM } \\
(\mathrm{deg})\end{array}$ & $\begin{array}{l}\text { SR+/- } \\
(\mathrm{V} / \mu \mathrm{s})\end{array}$ & $\begin{array}{l}\text { Area } \\
\left(\mathrm{mm}^{2}\right)\end{array}$ & $\begin{array}{l}\text { Supply } \\
(\mathrm{V})\end{array}$ & $\begin{array}{l}\text { Power } \\
(\mu \mathrm{W})\end{array}$ & FOM \\
\hline 1998 & 2 & 22 & 1.3 & 48.8 & 57 & $0.7 / 1.6$ & 1.51 & 1 & 287 & 10 \\
\hline 2000 & 1.2 & 15 & 1.9 & 87 & 61 & $0.8 / 1$ & 0.81 & 1 & 410 & 13.7 \\
\hline 2001 & 0.5 & - & 2 & $62-69$ & 57 & 0.5 & - & 1 & 40 & 50 \\
\hline 2002 & 2.5 & - & 5.6 & 79 & 62 & - & 0.5 & 0.9 & 0.45 & 13.4 \\
\hline 2003 & 0.25 & 18 & 1.2 & 52 & 60 & 0.2 & 0.006 & 0.8 & 8 & 218 \\
\hline $\begin{array}{l}2005 \\
(\text { Bulk })\end{array}$ & 0.18 & 20 & 2.5 & 52 & - & 2.89 & 0.026 & 0.5 & 110 & 22.7 \\
\hline $\begin{array}{l}2005 \\
(\mathrm{Gate})\end{array}$ & 0.18 & 20 & 10 & 62 & - & 2 & 0.017 & 0.5 & 75 & 133.4 \\
\hline 2009 & 0.35 & 2.5 & 540 & 62 & 52 & - & - & 0.9 & 9.1 & 12.3 \\
\hline 2012 & 0.35 & 15 & 11.67 & 88.3 & 66.1 & $2.53 / 1.37$ & 0.157 & 1 & 197 & 88.9 \\
\hline
\end{tabular}

\section{Quasi-Floating Gate MOSFET}

Quasi-Floating gate is similar to Floating gate MOSFET but except that gate weakly connected to one of the supply rail via a very high value resistor. Advantage of QFGMOS over FGMOS is that it reduces the charge accumulation problem.

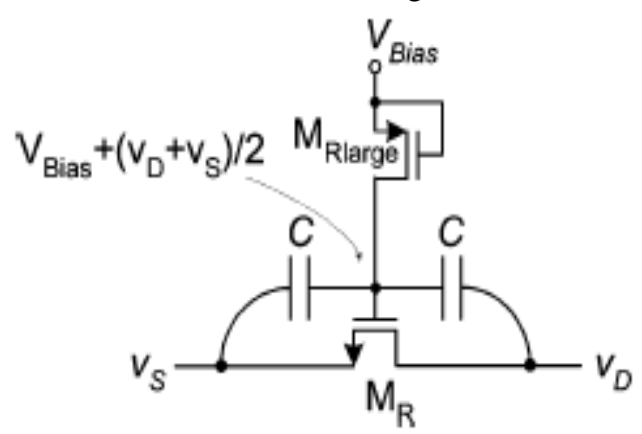

Fig-3: Triode transistor with enhanced linearity using QFG Technique [8]

Fig-3 express that how QFGMOS technique improve the linearity term when MOS operates in triode region. Here two input QFGMOS transistor in triode region act as active resistor [8].

\section{Dynamic Threshold MOS (DTMOS)}

In this technique both gate and body terminal are tied together. DTMOS concept is used when gate and body voltages are raised then it helps to minimize the threshold voltage of the device. Threshold voltage helps to reduce the power consumption [9]. DTMOS plays excellent role for increasing current drive and trans-conductance value. Disadvantage of this DTMOS concept is large junction capacitance, due to large capacitive value area of the device enhanced

\section{E. MOSFET Operating in Sub-threshold/ Weak inversion Region}

In past more focus was on strong inversion region but weak inversion is also important for low power CMOS analog circuit design [10] It is very useful in controlling threshold voltage and reducing power consumption. Advantage of this technique is that it helps to enhance $g_{m} / I_{d}$ value which is new trending technique as compare to current square law method. Due to week inversion trans-conductance value increased and helps to improve amplification of MOSFET. Drawback of week inversion is that it is not beneficial for very high frequencies applications

\section{CIRCUIT PERFORMANCE}

Published By: 


\section{IMPROVEMENT TECHNIQUES}

Circuit performance of analog and mixed circuits depends upon its essential parameters like DC gain, slew rate and bandwidth. These techniques are helpful to enhance the performance of analog circuits. These are as follows:

\section{A. Flipped Voltage Follower}

Flipped voltage follower is a cascade amplifier with negative feedback circuit. This circuit has high current sinking capability due to low output impedance [11]. This technique is very helpful for low static power dissipation, low distortion and unity voltage gain. High slew rate, ICMR and large voltage swing are useful for enhancing device performance. Voltage buffer circuit is able to sink large amount of current from load but loop hole of this circuit is that its sourcing capabilities is limited whereas source follower circuit has input current held constant value and it is independent of output current. This is also called voltage follower with shunt feedback.

\section{B. Differential Flip Voltage Followers (DFVF)}

Differential flip voltage follower is built by the help of FVF after adding one more MOS.[11] In DFVF maximum output current can be very high than quiescent current. In this technique output can be available as both current as well as voltage form. Fig-4(a) is called as FVF Pseudo differential pair. It is enhance version of DFVF by adding extra one transistor. Fig-4(b) shows DC output current vs. differential voltage graph. Main difference between DFVF and FVFDP is that output current is large in case of DFVF when the differential output voltage should be positive and in case of FVFDP differential voltage should be negative.

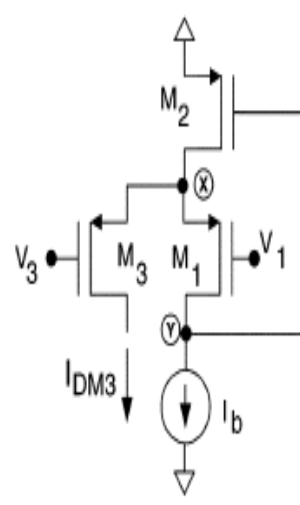

(a)

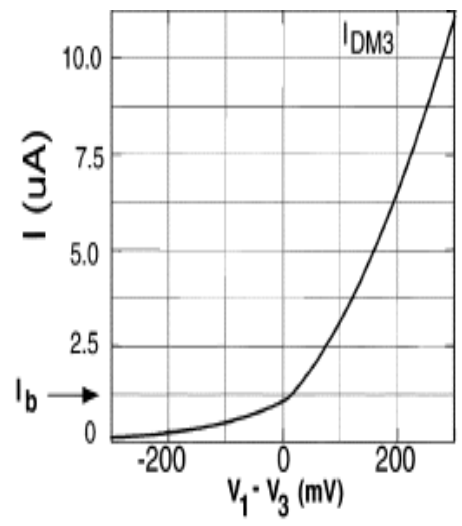

(b)
Fig-4: (a) FVFDP (b) DC transfer characteristic [11]

\section{Fully Differential Flip Voltage Follower (FDFVF)}

Fully differential voltage follower technique is using two DFVF to find the behavior of fully differential circuit. This circuit similar to DFVF very high output current can occur as compare to quiescent current and advantage of this circuit is that it can work on low supply voltage.

\section{Supper Source Follower (SSF)}

FVF technique is very useful for performance aspects of analog circuit but there few drawback of this circuit like small voltage swing, limited input voltage range. To reduce the loop hole of previous circuit Source follower circuit comes into picture. It is very useful as voltage buffer and level shifter [12]. SSF circuit has negative feedback which is very good for making device stable. Better current efficiency, less distortion, very high linearity and no restrictions on input ranges are positive side of super source follower circuit. SSF express when input voltage is constant output voltage increases.

\section{E. Self Cascode(SC) Structure}

Self cascode is two transistor based structure and it is very beneficial to provide higher output impedance and consumes very less area [13]. It has very high cut-off frequency due to shorter physical length. Fig- 5 shows gain boosted self cascode amplifier stage and the gain of simple amplifier can be increased by amplifier circuit by increasing the cascoding effect of transistor $\mathrm{M}_{2}$ [13].

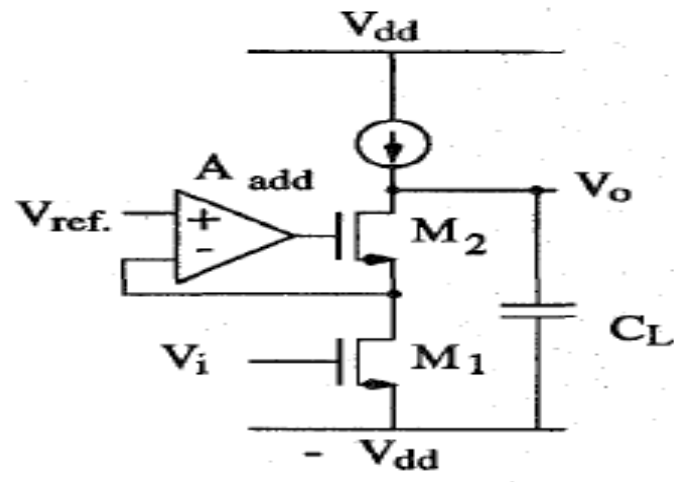

Fig-5: Gain boosted cascode amplifier stage [13]

\section{F. Feedback Circuits}

Feedback means some portion of output is return to input of the circuit [14]. In Fig-6 shows Common mode feedback circuit. CMFB is used to control the stability of the device and make device more stable. Recycling and reusing technique is similar to feedback technique but here same circuit or device is used for additional task [15].

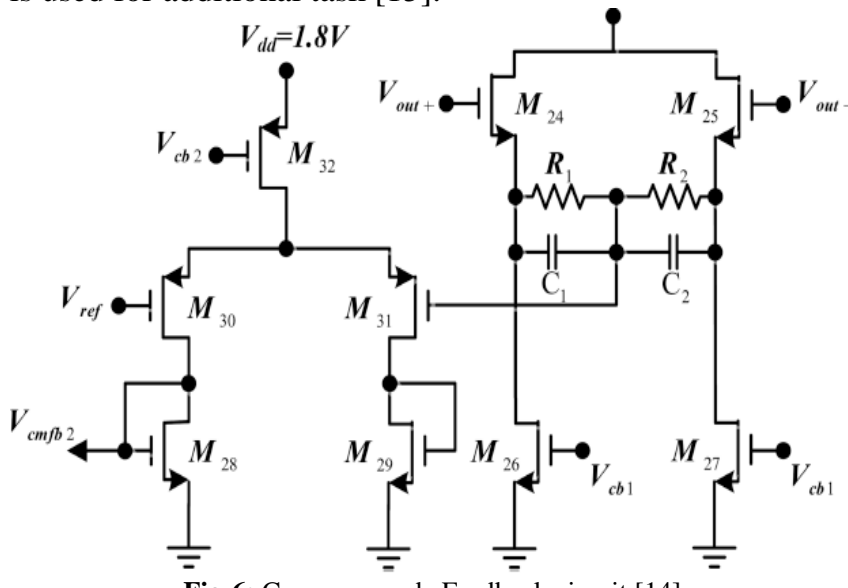

Fig-6: Common mode Feedback circuit [14]

\section{G. Folded Cascode Amplifier}

Folded cascode is very essential circuit for high gain and high output resistance. When folded cascode is used in place of self cascode then power dissipation is 
minimized [16]. It is mostly used in analog circuits. Fig-7 shows conventional folded cascode circuit which helps to reduce the power consumption. In two- stage operational amplifier first stage differential amplifier is replaced by folded cascode circuit for high amplification purpose.

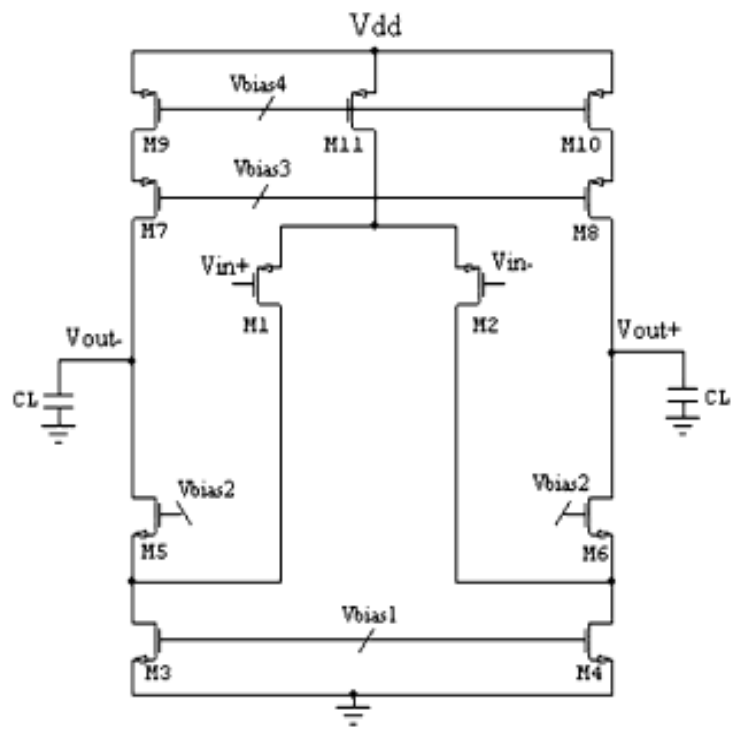

Fig-7: Conventional Folded cascode [16]

\section{H. Resistance Compensation Technique \& Current Enhancer Technique}

Current enhancer or current booster technique is helpful for current enhancement purpose and current is increased by the help of external transistor which is connected parallel side to regulate the circuit [17]. Resistance compensation technique is a method in which, compensate the resistance value inside the circuit accordingly and increase the performance of the circuit [18]. Fig-8 shows state diagram of RC-PLBC which helps PLL (Phase locked loop) circuit for frequency tracking, Phase tracking and phase locked purpose [18].

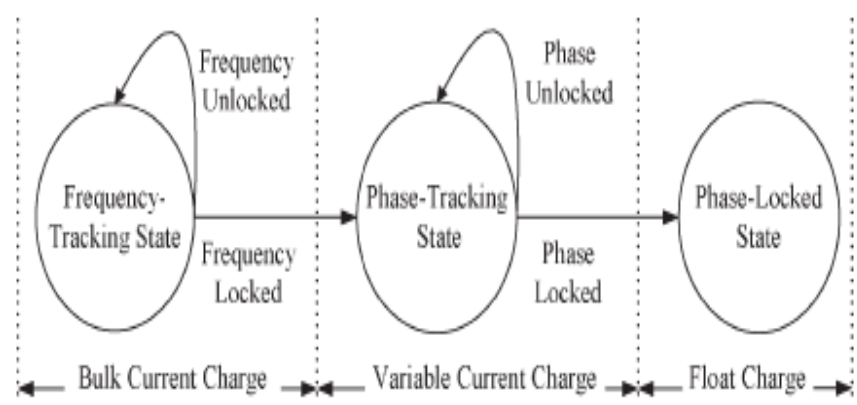

Fig-8: Equivalent state diagram of RC-PLBSC [18]

\section{VARIOUS BUILDING BLOCKS OF ANALOG CIRCUITS}

These are the basic building blocks of analog and mixed circuits and exceptionally basic in structuring any analog circuit based device. Operational-amplifier is a basic building block of any analog device and it is a three terminal gadget and performs all mathematical operation and device formation. OTA is operational transconductance amplifier. It is very versatile and dominating block in analog domain [19].
All the circuit which is based on signal processing concept is designed by OTA.

MI-OTA basically OTA design with multiple input terminals and it converts all input signals to a single output current [18] Transconductance value is increased and it will help for amplification purpose. CC stands for current conveyors and it is an alternative of op-amp in analog circuit. It gives high gain over large bandwidth and useful for high gain bandwidth product, speed of the device enhanced when GBW is large [1]-[5]. VDTA is voltage difference transconductance amplifier provides differential input voltage and it multiplied to transconductance results provide high current at output terminal. VDTA is designed by the help of two OTA in cascade form. VDTA is beneficial in terms of low power, high input impedance, high transconductance and high bandwidth. CDTA stands for current differencing Transconductance amplifier and it combines the merit of both the circuit CC and OTA [19]. High slew rate, absence of parasitic capacitance and simple design are advantage of CDTA circuit.

\section{CONCLUSION}

Analog circuit is most important part of the all gadgets. This paper focused to describe various power supply reduction method and performance improvement strategies. This will help to improve the performance of continuous signals. Floating gate, DTCMOS, recycling technique and feedback circuits are excellent technique for designers to achieve high slew rate, high gain and high bandwidth to make device performance excellent. There are bunches of good work remain for new comers in analog domain. EDA tools are also available in the market which helps to reduce the complexity of analog circuit during research.

\section{REFERENCES}

[1] B. Razavi, "Design of Analog CMOS Integrated Circuits", $27^{\text {th }}$ ed. New Delhi: McGraw Hill Education (India) Private Limited, 2013, pp 291-339.

[2] R. Baker, "CMOS Circuit Design, Layout and simulations", $2^{\text {nd }}$ ed. New Delhi: Wiley-Blackwell (an imprint of john Wiley \& Sons), 2010, pp. 773-792.

[3] P. Allen and D. Holberg, "CMOS Analog Circuit Design", $2^{\text {nd }}$ ed. New Delhi: Oxford University Press, USA, 2011, pp. 72-87.

[4] A. Sedra and K. smith, Microelectronics circuits, $5^{\text {th }}$ ed. New Delhi: Oxford University Press, 2004, pp. 287-297.

[5] Paul R. Gray, Robert G. Mayer, "Analysis and design of analog integrated circuits", (John Wiley and sons) $5^{\text {th }}$ ed. 08-043583, 2001

[6] Benjamin J. Blalock and Phillip E. Allen, "A Low-Voltage, Bulk-Driven MOSFET Current Mirror for CMOS Technology", IEEE 0-7803-2570-2/95 1995.

[7] Liang Zuo, Syed K. Islam, "Low-Voltage Bulk-Driven Operational Amplifier With Improved Transconductance ”, IEEE tran. Of circuit \& system Vol-60 2012

[8] Jose Maria Algueta Miguel, Antonio J. Lopez-Martin, Lucia Acosta, "Using Floating Gate and Quasi-Floating Gate Techniques for Rail-to-Rail Tunable CMOS Transconductor Design",IEEE trans. Of circuit \& system Vol.58 2011.

[9] Fariborz Assaderaghi et.al , "Dynamic Threshold-Voltage MOSFET (DTMOS) for Ultra low voltage VLSI", IEEE trans. On electronic devices Vol.44 No.3, 1997.

[10] Francesco Forti and Michael E. Wright, "Measurement of MOS Current Mismatch in the Weak Inversion Region", IEEE Journal of solid state circuits Vol. 29, 1994. 
[11] Ramón González Carvajal, Jaime Ramírez-Angulo, Antonio J. López-Martín, Antonio Torralba, Juan Antonio Gómez Galán, Alfonso Carlosena, Fernando Muñoz Chavero, "The Flipped Voltage Follower: A Useful Cell for Low-Voltage Low-Power Circuit Design" IEEE trans. On circuit \& system, Vol.52, 2005.

[12] Mr. D. K. Shedge1, Mr. D. A. Itole2, Mr. M. P. Gajare3, and Dr. P. W Wani4, "Analysis and Design of CMOS Source Followers and Super Source Follower", ACEEE Int. J. on Control System and Instrumentation, Vol. 4, No. 2, June 2013.

[13] Pradip Mandal, V. Viisvanathan, "A Self-Biased High Performance Folded Cascode CMOS Op-Amp”, IEEE conference on VLSI design 0-8186-7755-4/96, 1996

[14] Sayed Mahmoud Anisheh, Hossein Shansi, Mitra Mirhassani, "Positive feedback technique and split length transistors for DC-gain enhancement of two stage op-amp", IET circuits devices syst., Vol.11, Iss-6, 2017, pp. 605-612.

[15] Meysam Akbari, Sadegh Biabanifard, Shahrouz Asadi, Mustapha C.E. Yagoub, "Design and analysis of DC gain and transconductance boosted recycling folded cascode OTA", AEU ELSEVIER, 10.1016, 2014

[16] Mahdieye Pourabdollah, "A new gain-enhanced and slew-rate-enhanced folded-cascode opAmp",Springer Science+Business Media New York 2016.

[17] Elad Alon, Jaeha Kim, Sudhakar Pamarti, Ken Chang, Mark Horowitz “ Replica Compensated Linear Regulators for Supply-Regulated Phase-Locked Loops", IEEE Journal of solid state Vol.41 2006

[18] Liang-Rui Chen, Chuan-Sheng Liu, Jin-Jia Chen, "Improving Phase-Locked Battery Charger Speed by Using Resistance-Compensated Technique", IEEE trans. On Industrial Electronics Vol-56, No.4 2009

[19] Neha Gupta1, Sapna Singh2, Meenakshi Suthar3 and Priyanka Soni4, "LOW POWER LOW VOLTAGE BULK DRIVEN BALANCED OTA", International Journal of VLSI design \& Communication Systems (VLSICS) Vol.2, No.4, December 2011

\section{AUTHORS PROFILE}

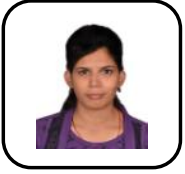

Saumya Srivastava has achieved her B.Tech degree in Electronics \& Communication Engineering degree from Dr. A.P.J. Abdul Kalam Technical University, Lucknow, Uttar Pradesh. She has completed her summer intern in VLSI circuit Fabrication area at Semiconductor Labotary department of space India, Mohali.. Now she is M.E. Scholar in the field of VLSI Designing and her one paper published in ACM ICPS series.

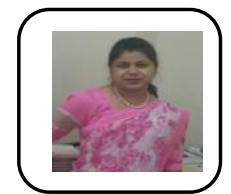

Dr. Tripti Sharma has achieved her M.Tech. and $\mathrm{Ph}$. D. degree in the field of Low Power VLSI Circuits Design. She has more than 16 years of teaching experience along with intense research interest. After stepping into professional world, she started her career as lecturer with C.S.J.M University (Govt.), Kanpur and continued it up to late 2003. after that she served Mody Institute of Technology and Science (Deemed University), Rajasthan and stayed for more than a decade. There she worked in the core team to achieve NBA, AICTE and NAAC accreditation. Later on she joined Vivekananda Global University in 2015 as Professor and Head of the ECE deptt. and along with all the academic \& administrative responsibilities she worked there for getting UGC affiliation for the University in her short stay. She joined Chandigarh University as Professor of the ECE department in January 2016. Her research interests include Digital \& Analog low power VLSI circuits and Double Gate MOSFET Circuit Design \& Analysis. She has more than 60 publications in International Journals and National/ International Conferences in the areas of high-performance integrated circuits and emerging semiconductor Technologies. She has also authored 05 technical books useful for research in the field of digital circuit design and filed a patent for the neurodevelopmental disorder to help the society. 